\title{
GRB 091127: The cooling break race on magnetic fuel ${ }^{\star}$
}

\author{
R. Filgas ${ }^{1}$, J. Greiner ${ }^{1}$, P. Schady ${ }^{1}$, T. Krühler ${ }^{1,2,3}$, A. C. Updike ${ }^{4,5,6}$, S. Klose ${ }^{7}$, M. Nardini ${ }^{1, \star \star}$, D. A. Kann ${ }^{7}$, A. Rossi ${ }^{7}$, \\ V. Sudilovsky ${ }^{1}$, P. M. J. Afonso ${ }^{1, \star \star \star}$, C. Clemens ${ }^{1}$, J. Elliott ${ }^{1}$, A. Nicuesa Guelbenzu ${ }^{7}$, F. Olivares E. ${ }^{1}$, and A. Rau ${ }^{1}$ \\ 1 Max-Planck-Institut für extraterrestrische Physik, Giessenbachstraße 1, 85748 Garching, Germany \\ e-mail: filgas@mpe.mpg.de \\ 2 Universe Cluster, Technische Universität München, Boltzmannstraße 2, 85748 Garching, Germany \\ 3 Dark Cosmology Centre, Niels Bohr Institute, University of Copenhagen, Juliane Maries Vej 30, 2100 Copenhagen, Denmark \\ 4 Department of Physics and Astronomy, Clemson University, Clemson, SC 29634-0978, USA \\ 5 CRESST and the Observational Cosmology Laboratory, NASA/GSFC, Greenbelt, MD 20771, USA \\ 6 Department of Astronomy, University of Maryland, College Park, MD 20742, USA \\ 7 Thüringer Landessternwarte Tautenburg, Sternwarte 5, 07778 Tautenburg, Germany
}

Received 13 July 2011 / Accepted 13 September 2011

\begin{abstract}
Aims. Using high-quality, broad-band afterglow data for GRB 091127, we investigate the validity of the synchrotron fireball model for gamma-ray bursts (GRBs), and infer physical parameters of the ultra-relativistic outflow.

Methods. We used multi-wavelength (NIR to X-ray) follow-up observations obtained with GROND simultaneously in the $g^{\prime} r^{\prime} i^{\prime} z^{\prime} J H$ filters and the XRT onboard the Swift satellite in the 0.3 to $10 \mathrm{keV}$ energy range. The resulting afterglow light curve is of excellent accuracy with relative photometric errors as low as 1\%, and the spectral energy distribution (SED) is well-sampled over 5 decades in energy. These data present one of the most comprehensive observing campaigns for a single GRB afterglow and allow us to test several proposed emission models and outflow characteristics in unprecedented detail.

Results. Both the multi-color light curve and the broad-band SED of the afterglow of GRB 091127 show evidence of a cooling break moving from high to lower energies. The early light curve is well described by a broken power-law, where the initial decay in the optical/NIR wavelength range is considerably flatter than at X-rays. Detailed fitting of the time-resolved SED shows that the break is very smooth with a sharpness index of $2.2 \pm 0.2$, and evolves towards lower frequencies as a power-law with index $-1.23 \pm 0.06$. These are the first accurate and contemporaneous measurements of both the sharpness of the spectral break and its time evolution. Conclusions. The measured evolution of the cooling break $\left(v_{\mathrm{c}} \propto t^{\sim-1.2}\right)$ is not consistent with the predictions of the standard model, wherein $v_{\mathrm{c}} \propto t^{\sim-0.5}$ is expected. A possible explanation for the observed behavior is a time dependence of the microphysical parameters, in particular the fraction of the total energy in the magnetic field $\epsilon_{\mathrm{B}}$. This conclusion provides further evidence that the standard fireball model is too simplistic, and time-dependent micro-physical parameters may be required to model the growing number of well-sampled afterglow light curves.
\end{abstract}

Key words. ISM: jets and outflows - X-rays: bursts - X-rays: individuals: GRB 091127

\section{Introduction}

Gamma-ray bursts (GRBs) are among the most energetic explosions in the universe. The leading model for their afterglows is the synchrotron fireball (Mészáros \& Rees 1997; Piran 1999; Mészáros 2002; Zhang \& Mészáros 2004). In this model, the afterglow arises from the synchrotron emission of shockaccelerated electrons in a fireball interacting with the circumburst medium. The spectral energy distribution (SED) of such emission is well modeled by several broken power-laws connected at characteristic break frequencies (Sari et al. 1998). The model predicts a break in the light curve when the cooling frequency $\left(v_{c}\right.$, the frequency of electrons whose radiative cooling time-scale equals the dynamical time of the system) or the

^ Tables 3 and 4 are only available at the CDS via anonymous ftp to cdsarc.u-strasbg.fr $(130.79 .128 .5)$ or via http://cdsarc.u-strasbg.fr/viz-bin/qcat?J/A+A/535/A57

$\star \star$ Present address: Università degli studi di Milano-Bicocca, Piazza della Scienza 3, 20126, Milano, Italy.

$\star \star \star$ Present address: American River College, Physics \& Astronomy Dpt., 4700 College Oak Drive, Sacramento, CA 95841, USA. characteristic synchrotron frequency $\left(v_{\mathrm{m}}\right.$, peak frequency for the minimal energy of the radiating electrons) passes through the observed bands. Such breaks in the light curve have been, however, difficult to identify reliably as the passage of the above frequencies.

With the development of rapid-response telescopes and multi-wavelength instruments, we expected to detect the movement of the break frequencies. However, this movement has only possibly been observed directly in the afterglow of GRB 080319B (Racusin et al. 2008). Detections of the spectralbreak movements in other GRBs were mostly based on the evolution of the GRB afterglow light curves in just one or few filters, where the subtle steepening is visible and is attributed to the passage of the cooling frequency, for example GRB 990510 (Kumar \& Panaitescu 2000), GRB 030329 (Sato et al. 2004; Uemura et al. 2003), GRB 040924 (Huang et al. 2005), GRB 041218 (Torii et al. 2005), GRB 050408 (Kann et al. 2010), GRB 050502A (Yost et al. 2006), GRB 060729 (Grupe et al. 2010), etc. In some cases, this claim is supported by measured spectral evolution. Lipkin et al. (2004) measured the $B-R$ color change in the afterglow of GRB 030329, supporting 
the theory of the cooling break passage derived from the lightcurve steepening. Only very few GRBs had coverage in several bands good enough to model the evolution of the afterglow spectrum. In one such rare case, de Ugarte Postigo et al. (2005) modelled the broad-band SED of the afterglow of GRB 021004 at three distinct epochs, though only the low frequency part of the spectrum shows any evolution. In order to study such spectral evolutions in detail, continuous coverage with high signal-tonoise ratio in several bands simultaneously is required.

The Swift satellite (Gehrels et al. 2004) makes it possible to study the afterglow emission starting at very early times thanks to its rapid slewing capability, a precise localization of GRBs with its Burst Alert Telescope (BAT, Barthelmy et al. 2005), and early follow-up with onboard telescopes sensitive at X-ray (XRT, Burrows et al. 2005) and ultraviolet/optical (UVOT, Roming et al. 2005) wavelengths. Since its launch in 2004, Swift has provided many early and well-sampled afterglow light curves and X-ray spectra. Blustin et al. (2006) for example fitted broad-band SEDs of the afterglow of GRB 050525A with a cooling break between early optical and X-ray data and with a simple power-law through later epochs, suggesting a spectral evolution. However, such sudden spectral change can sometimes be also attributed to another component with a different electron distribution present in the emission at later times (Filgas et al. 2011).

The most convincing measurement of the cooling break movement to this date is the case of the naked-eye burst GRB 080319B (supplementary information in Racusin et al. 2008; Schady et al., in prep.). Due to the enormous brightness of this event, these authors were able to fit broad-band SEDs at several epochs using Swift UVOT and XRT data, as well as a multitude of optical and NIR ground-based data, showing a clear temporal evolution of a break that may be attributed to the cooling break. The previously mentioned examples show that in case of regularly bright GRB afterglows small telescopes cannot provide the accuracy needed for such detailed study.

The Gamma-Ray burst Optical Near-infrared Detector (GROND, Greiner et al. 2008; Greiner et al. 2007) at the $2.2 \mathrm{~m}$ MPI/ESO telescope at La Silla observatory is capable of providing high-quality, very well-sampled data in seven bands simultaneously and therefore opening a new region with respect to data quality and quantity. Such high-precision data allow not only for a detailed study of afterglow light curves (Greiner et al. 2009b; Nardini et al. 2011) but also jets of GRBs (Krühler et al. 2009), the dust in their host galaxies (Krühler et al. 2008; Küpcü Yoldaş et al. 2010; Greiner et al. 2011; Krühler et al. 2011b), their redshifts (Greiner et al. 2009a; Krühler et al. 2011a) and much more.

Here we provide details of the Swift and GROND observations of the afterglow of GRB 091127 and discuss the light curves and SEDs in the context of the fireball model thanks to very good energy coverage and sampling of our high-quality data. Throughout the paper, we adopt the convention that the flux density of the GRB afterglow can be described as $F_{v}(t) \propto t^{-\alpha} v^{-\beta}$, where $\alpha$ is the temporal and $\beta$ the spectral index. Unless stated otherwise in the text, all reported errors are at $1 \sigma$ confidence level.

\section{Observations}

\subsection{Prompt emission}

At $T_{0}=23: 25: 45 \mathrm{UT}$, the Swift/BAT was triggered by the long GRB 091127 (Troja et al. 2009). Due to an Earth-limb observing constraint, Swift could not slew to the target until $53 \mathrm{~min}$ after the trigger (Immler \& Troja 2009). The mask-weighted light curve shows three main peaks from $T_{0}-0.3$ to $T_{0}+10 \mathrm{~s}$, peaking at $\sim T_{0}, T_{0}+1.1 \mathrm{~s}$ and at $T_{0}+7 \mathrm{~s}$. The measured $T_{90}$ $(15-350 \mathrm{keV})$ is $7.1 \pm 0.2 \mathrm{~s}$ (Stamatikos et al. 2009). The BAT prompt emission spectrum from $T_{0}-0.4$ to $T_{0}+7.5 \mathrm{~s}$ is best fitted using a simple power-law model with photon index $2.05 \pm 0.07$ and the total fluence in the $15-150 \mathrm{keV}$ energy range is $(9.0 \pm 0.3) \times 10^{-6} \mathrm{erg} \mathrm{cm}^{-2}$ (Stamatikos et al. 2009). We can get a better picture of the prompt emission from the instruments with larger energy coverage. Konus-Wind observed the burst in the $20 \mathrm{keV}-2 \mathrm{MeV}$ energy range and measured a fluence of $(1.22 \pm 0.06) \times 10^{-5} \mathrm{erg} \mathrm{cm}^{-2}$. The time-integrated spectrum of the burst (from $T_{0}$ to $T_{0}+8.4 \mathrm{~s}$ ) is well fitted by a power-law with exponential cutoff model with $\alpha=-1.95 \pm 0.10$, and $E_{\text {peak }}=21.3_{-3}^{+4} \mathrm{keV}$ (Golenetskii et al. 2009). Using a standard concordance cosmology $\left(H_{0}=71.0 \mathrm{~km} \mathrm{~s}^{-1} \mathrm{Mpc}^{-1}, \Omega_{\mathrm{M}}=\right.$ $0.27, \Omega_{\Lambda}=0.73$, Komatsu et al. 2009), and a redshift of $z=0.49$ (Cucchiara et al. 2009; Thöne et al. 2009), we calculate the bolometric $(1 \mathrm{keV}-10 \mathrm{MeV})$ energy release of GRB 091127 to be $E_{\text {iso }}=1.4 \times 10^{52} \mathrm{erg}$. Fermi GBM provides even better energy coverage and the obtained time-averaged spectrum from $T_{0}+0.002 \mathrm{~s}$ to $T_{0}+9.984 \mathrm{~s}$ is adequately fit by a Band function (Band et al. 1993) with $E_{\text {peak }}=35.5 \pm 1.5 \mathrm{keV}, \alpha_{\text {prompt }}=-1.26 \pm$ 0.07 , and $\beta_{\text {prompt }}=-2.22 \pm 0.02$. The event fluence in the $8-1000 \mathrm{keV}$ energy range in this time interval is $(1.92 \pm 0.02) \times$ $10^{-5} \mathrm{erg} \mathrm{cm}^{-2}$ (Goldstein et al., in prep.). This results in the bolometric energy release of $E_{\text {iso }}=1.6 \times 10^{52} \mathrm{erg}$, making GRB 091127 consistent within $2 \sigma$ with the most updated Amati $E_{\text {peak }}-E_{\text {iso }}$ relation (Amati et al. 2002).

\subsection{Swift $X R T$}

The Swift/XRT started observations of the field of GRB 091127 53 min after the trigger (Evans et al. 2009b). The XRT light curve and spectra were obtained from the XRT repository (Evans et al. 2007, 2009a). Spectra were grouped using the grppha task and fitted with the GROND data in XSPEC v12 using $\chi^{2}$ statistics. The combined optical/X-ray SEDs were fitted with power-law and broken power-law models and two absorbing columns: one Galactic foreground with a hydrogen column density of $N_{\mathrm{H}}=2.8 \times 10^{20} \mathrm{~cm}^{-2}$ (Kalberla et al. 2005) and another one that is local to the GRB host galaxy at $z=0.49$ (Cucchiara et al. 2009; Thöne et al. 2009). Only the latter was allowed to vary in the fits. To investigate the dust reddening in the GRB environment, the zdust model was used, which contains Large and Small Magellanic Clouds (LMC, SMC) and Milky Way (MW) extinction laws from Pei (1992). The errors of the broad-band SED fits on any single parameter were obtained using the uncert command in XSPEC. This calculates the error on the parameter in question while allowing all the other non-frozen parameters in the model to vary.

\subsection{GROND}

GROND responded to the Swift GRB alert and initiated automated observations at 00:24 UT, $58 \mathrm{~m}$ after the trigger (Updike et al. 2009). GROND imaging of the field of GRB 091127 continued for ten further epochs, the last being acquired on October 31st, 2010. Due to the broken chip of the NIR $K$-band detector, there are no data available for this filter. A variable point source was detected in all other bands by the automated GROND pipeline (Küpcü Yoldaş et al. 2008). The position of the transient was calculated to be RA $(\mathrm{J} 2000)=02: 26: 19.87$ 


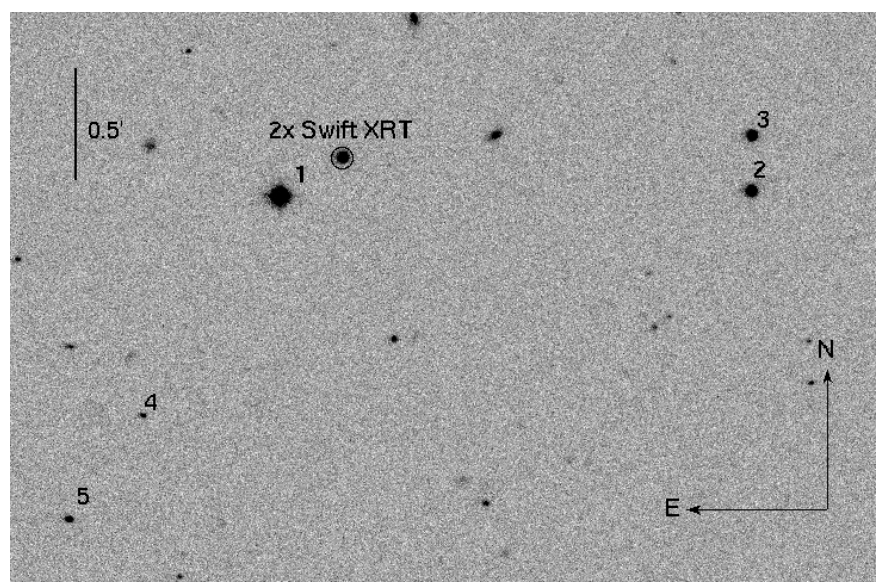

Fig. 1. GROND $r^{\prime}$ band image of the field of GRB 091127 obtained $4.3 \mathrm{ks}$ after $T_{0}$. The optical afterglow is visible inside the Swift XRT error circle with double diameter for better clarity. The secondary standard stars are numbered from 1 to 5 and their magnitudes reported in Table 5.

and Dec $(\mathrm{J} 2000)=-18: 57: 08.6$ compared to USNO-B reference field stars (Monet et al. 2003) with an astrometric uncertainty of 0.3 .

The optical and NIR image reduction and photometry were performed using standard IRAF tasks (Tody 1993) similar to the procedure described in detail in Krühler et al. (2008). A general model for the point-spread function (PSF) of each image was constructed using bright field stars and fitted to the afterglow. In addition, aperture photometry was carried out, and the results were consistent with the reported PSF photometry. All data were corrected for a Galactic foreground reddening of $E_{B-V}=$ 0.04 mag in the direction of the burst (Schlegel et al. 1998), corresponding to an extinction of $A_{V}=0.12$ using $R_{V}=3.1$, and in the case of $J H$ data, transformed to $\mathrm{AB}$ magnitudes.

Optical photometric calibration was performed relative to the magnitudes of five secondary standards in the GRB field, shown in Fig. 1 and Table 5. During photometric conditions, a spectrophotometric standard star SA94-242, a primary SDSS standard (Smith et al. 2002), was observed within a few minutes of observations of the GRB field. The obtained zeropoints were corrected for atmospheric extinction and used to calibrate stars in the GRB field. The apparent magnitudes of the afterglow were measured with respect to the secondary standards reported in Table 5. The absolute calibration of $J H$ bands was obtained with respect to magnitudes of the Two Micron All Sky Survey (2MASS) stars within the GRB field obtained from the 2MASS catalog (Skrutskie et al. 2006). All data are listed in Tables 3 and 4.

\section{Results}

\subsection{Afterglow light curve}

The X-ray light curve (Fig. 2) of the afterglow of GRB 091127 is best fitted with a smoothly broken power-law model (Beuermann et al. 1999) with an initial decay slope $\alpha_{\mathrm{X}}=1.02 \pm 0.04$, a time of the break at around $33 \mathrm{ks}$ and a post-break temporal slope of $1.61 \pm 0.04$ (Fig. 3, red. $\chi^{2}=1.03$, straight power-law has red. $\chi^{2}=1.80$, sharply broken power-law has red. $\chi^{2}=1.04$ ). The optical/NIR light curve follows the same model but with a much flatter initial temporal slope, which further flattens with increasing wavelength of GROND filters. Table 1 shows results

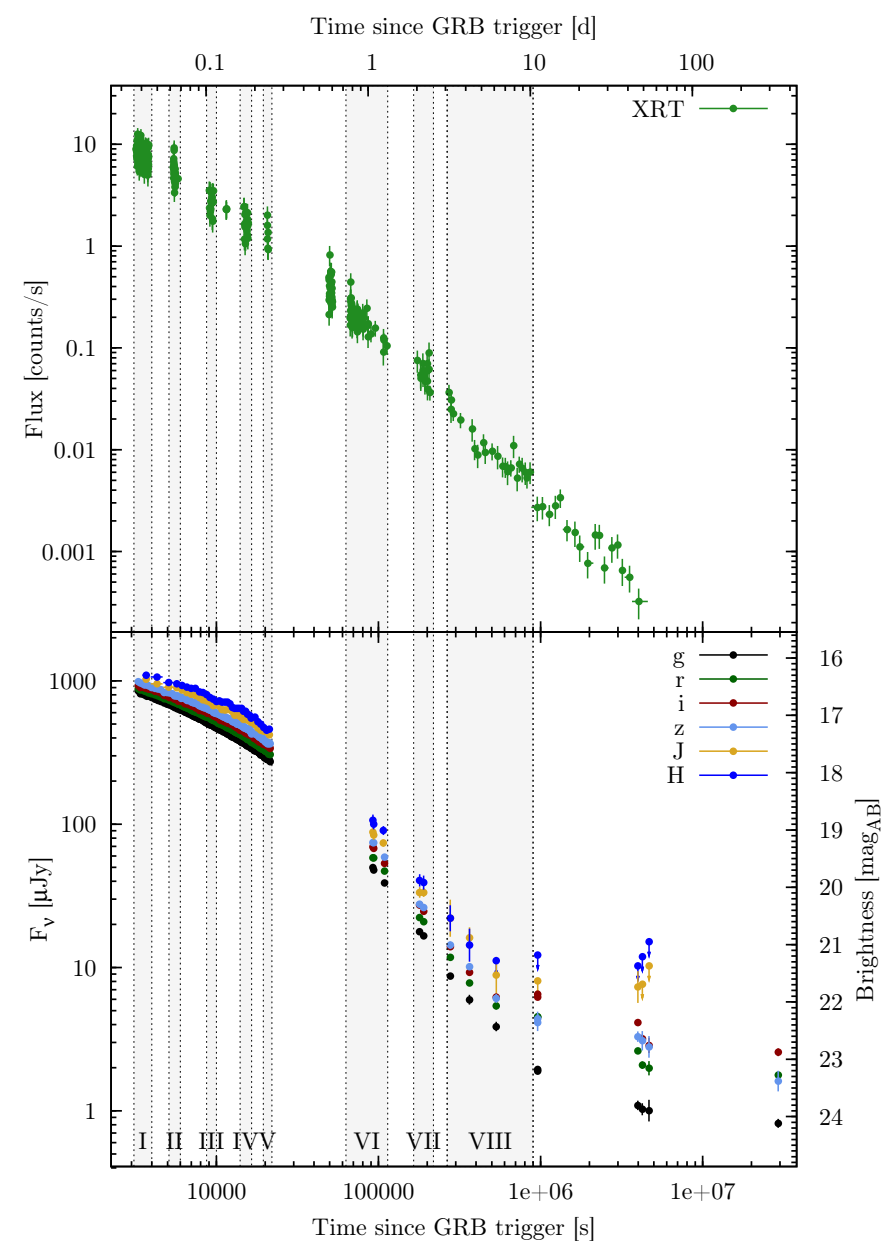

Fig. 2. Light curve of the X-ray (top panel) and GROND optical/NIR (bottom panel) afterglow of GRB 091127. Shown data are corrected for Galactic foreground extinction and are in $\mathrm{AB}$ magnitudes. Gray regions show the time intervals where broad-band SEDs were created (Fig. 5).

of the fitting of a smoothly broken power-law model to each band separately. The sharply broken power-law model provides a much worse fit with red. $\chi^{2}>10$ in the optical bands. This initial temporal slope is however difficult to measure because the pre-break optical/NIR data show a smooth curvature without a straight power-law segment. The reported temporal slope parameters fitted to these data should therefore be considered as estimates of power-law slopes of the earliest optical/NIR data.

The difference in the early decay between X-ray and optical/NIR wavelengths and among optical/NIR bands themselves suggest a strong color evolution, which we discuss in detail in the next section. The time of the X-ray break and the later decay index of the X-ray fit is within $1 \sigma$ errors of the fit to the optical bands and within $3 \sigma$ errors of the fit to the NIR bands. The optical/NIR data after $500 \mathrm{ks}$ are not fitted as they show contribution from the SN 2009nz bump described by Cobb et al. (2010), Berger et al. (2011) and Vergani et al. (2011). We did not subtract the SN magnitudes from the afterglow because this work is based mostly on the early data where the afterglow is dominant. Moreover, at even later times, the GROND decay after the break is consistent with the X-ray temporal slope, and the GROND SEDs are well-fitted with a straight power-law. We therefore argue that the influence of the emission not coming from the GRB itself is negligible throughout the time interval used for this study. 
Table 1. Light curve fit parameters for the afterglow of GRB 091127.

\begin{tabular}{lccccc}
\hline \hline Band & $\alpha_{1}$ & $t_{\text {break }}[\mathrm{s}]$ & $s$ & $\alpha_{2}$ & $\chi^{2} /$ d.o.f. \\
\hline XRT & $1.019 \pm 0.039$ & $33472 \pm 3349$ & $2.367 \pm 0.986$ & $1.605 \pm 0.038$ & $373 / 363$ \\
$g^{\prime}$ & $0.427 \pm 0.011$ & $33917 \pm 2047$ & $1.210 \pm 0.125$ & $1.687 \pm 0.050$ & $125 / 144$ \\
$r^{\prime}$ & $0.376 \pm 0.009$ & $29287 \pm 1195$ & $1.274 \pm 0.100$ & $1.557 \pm 0.033$ & $143 / 144$ \\
$i^{\prime}$ & $0.359 \pm 0.014$ & $30288 \pm 1671$ & $1.293 \pm 0.141$ & $1.532 \pm 0.042$ & $133 / 144$ \\
$z^{\prime}$ & $0.321 \pm 0.016$ & $32368 \pm 2295$ & $1.054 \pm 0.124$ & $1.609 \pm 0.056$ & $131 / 144$ \\
$J$ & $0.300 \pm 0.077$ & $24462 \pm 4453$ & $1.483 \pm 0.728$ & $1.396 \pm 0.147$ & $26 / 37$ \\
$H$ & $0.164 \pm 0.057$ & $21677 \pm 4310$ & $1.005 \pm 0.106$ & $1.417 \pm 0.068$ & $34 / 37$ \\
\hline
\end{tabular}

Notes. The temporal slopes have inaccuracies caused by a very smooth break, which reduces the number of datapoints used in the power-law slopes fitting. The fitting of the NIR bands is affected by the somewhat lower signal-to-noise ratio of the NIR data as compared to the optical bands.
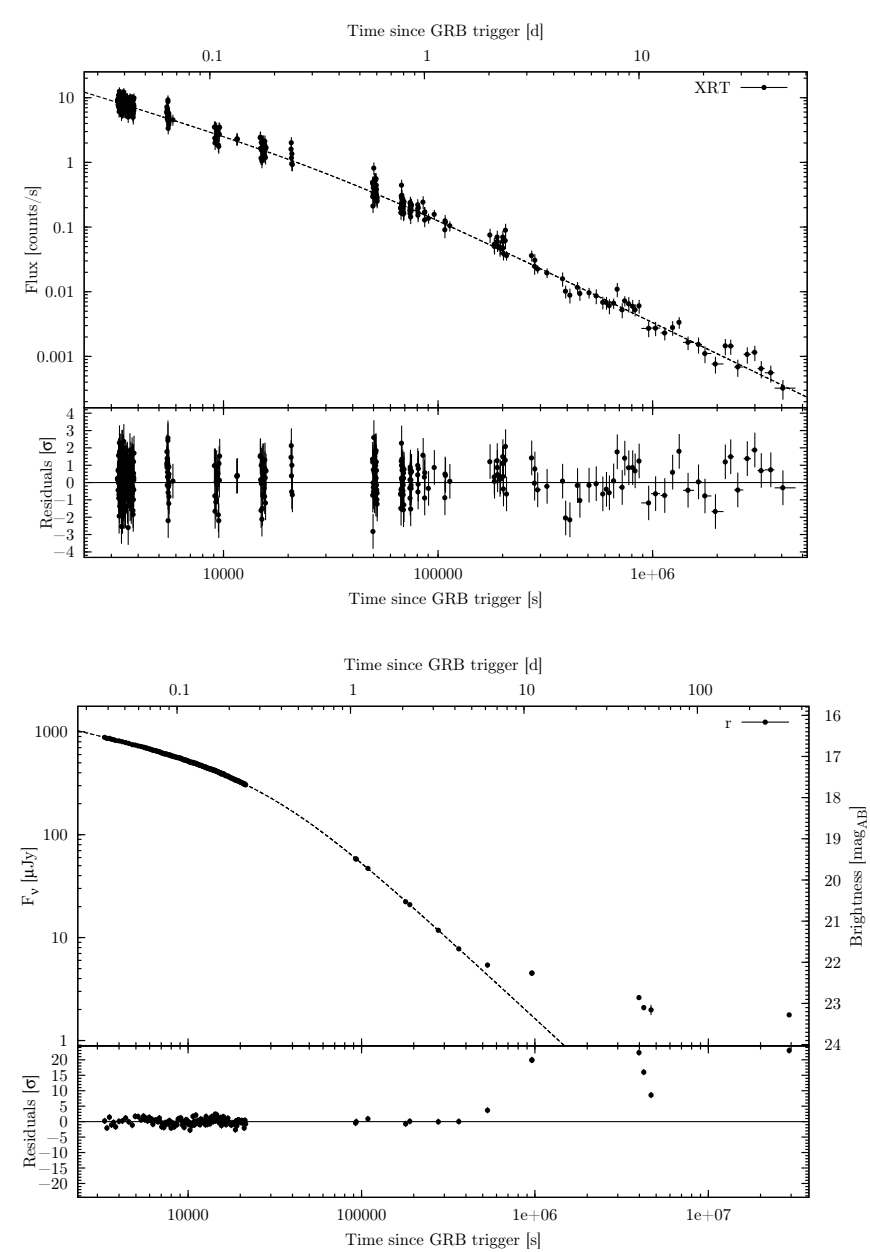

Fig. 3. The smoothly broken power-law fit to the X-ray light curve (top) and the GROND $r^{\prime}$ band data (bottom), the parameters of the fit are listed in Table 1. Residuals from the best-fit to the $r^{\prime}$ band data show the SN bump.

\subsection{Afterglow SEDs}

As already evident from the afterglow light curves, there is a strong spectral evolution in the optical/NIR wavelengths before the break. Thanks to the simultaneous multi-band observing capabilities of GROND, it is possible to measure the opti$\mathrm{cal} /$ NIR spectral slope as a function of time with high accuracy. Figure 4 shows that the optical/NIR spectral index rises from $0.23 \pm 0.04$ to $0.80 \pm 0.08$ between 3 and $300 \mathrm{ks}$. In addition, broad-band optical/NIR to X-ray SEDs were constructed at eight different time intervals within this period, which are indicated in the light curve (Fig. 2). Fits of optical/NIR data alone as well

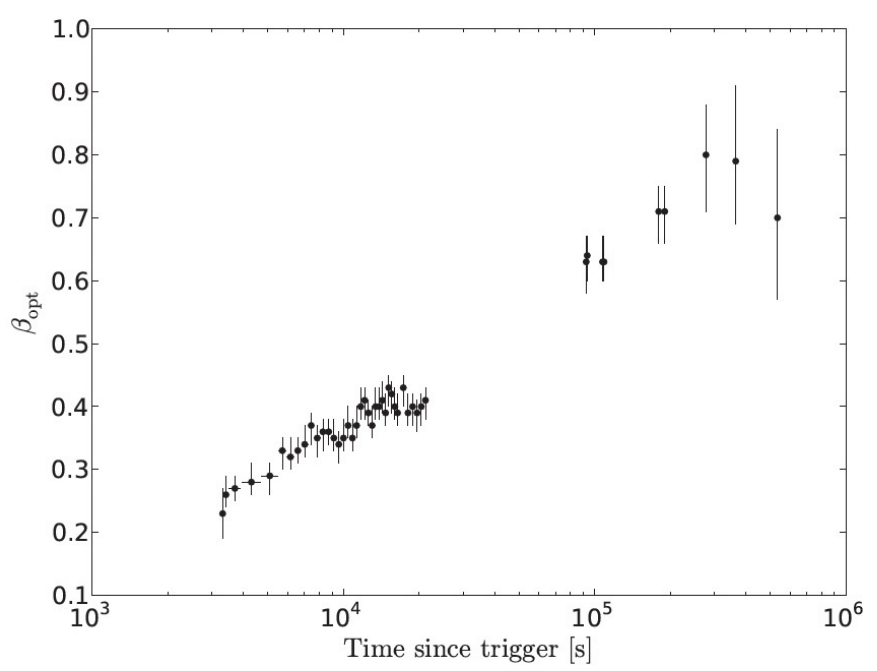

Fig. 4. The optical/NIR spectral slope as a function of time.

as the broad-band fits resulted in a host dust extinction that was consistent with zero, therefore in all the models we assumed no host dust extinction for simplicity.

Fitting the XRT-only spectrum using the full dataset we obtain the host absorbing column density $N_{\mathrm{H}}=(1.3 \pm 0.5) \times$ $10^{21} \mathrm{~cm}^{-2}$. Because the broad-band SEDs proved to be inconsistent with a simple power-law model, we used models that include a break between the X-ray and optical/NIR data. We initially fitted all eight epochs of broad-band SEDs simultaneously with a sharp broken power-law model, where the hostintrinsic absorbing column density and the X-ray spectral index are tied between each SED but left free to vary. The low energy spectral indices and energy of the break were left untied between SEDs and free to vary. The best fit (red. $\chi^{2}=1.11$ ) gives values of the host-equivalent neutral hydrogen density $N_{\mathrm{H}}=(3.2 \pm 0.6) \times 10^{20} \mathrm{~cm}^{-2}$ and the high-energy spectral index $\beta_{\mathrm{X}}=0.748 \pm 0.004$. The value of $N_{\mathrm{H}}$ is smaller than what we get using just the XRT data alone but is consistent within $2 \sigma$ with the one resulting from the XRT-only spectral fitting.

The best-fit optical parameters are listed in Table 2. This fit shows that the break evolves to larger wavelengths in time, through and beyond the optical/NIR bands (top panels of Fig. 5). The last two SEDs are consistent with a simple power-law continuum without any break. This is in agreement with the X-ray spectral index being within $1 \sigma$ errors consistent with optical/NIR-only spectral indices $0.71 \pm 0.04$ (at time of SED VII) and $0.80 \pm 0.08$ (at time of SED VIII). The temporal evolution of the break was fitted with a power-law $v_{\mathrm{c}} \propto t^{x}$ and the best-fit index was $x=-0.69 \pm 0.10$ (Fig. 5). 
R. Filgas et al.: GRB 091127: The cooling break race on magnetic fuel

Table 2. Best-fit parameters resulting from the sharp and smooth broken power-law fits to the broad-band SEDs.

\begin{tabular}{lcccc}
\hline \hline $\begin{array}{l}\text { SED } \\
\text { number }\end{array}$ & $\begin{array}{c}\text { Midtime [s] } \\
\text { of SED }\end{array}$ & $\begin{array}{c}\text { Low energy spectral } \\
\text { index using sharp break }\end{array}$ & $\begin{array}{c}\text { Cooling break [eV] } \\
\text { using sharp break }\end{array}$ & $\begin{array}{c}\text { Cooling break [eV] } \\
\text { using smooth break }\end{array}$ \\
\hline I & 3404 & $0.25_{-0.04}^{+0.02}$ & $29.9_{-5.5}^{+8.1}$ & $28.7_{-1.1}^{+1.1}$ \\
II & 5088 & $0.28 \pm 0.04$ & $22.6_{-4.2}^{+4.6}$ & $18.5_{-1.4}^{+1.5}$ \\
III & 9576 & $0.33_{-0.04}^{+0.03}$ & $13.9_{-2.7}^{+3.0}$ & $8.5_{-1.1}^{+1.2}$ \\
IV & 15135 & $0.41_{-0.03}^{+0.03}$ & $10.9_{-2.0}^{+2.9}$ & $4.4_{-0.5}^{+0.6}$ \\
V & 21193 & $0.39_{-0.03}^{+0.04}$ & $10.5_{-2.9}^{+4.4}$ & $4.3_{-0.9}^{+1.1}$ \\
VI & 107401 & $0.62_{-0.05}^{+0.04}$ & $2.6_{-0.6}^{+1.2}$ & $0.3_{-0.1}^{+0.2}$ \\
VII & 189939 & - & $<0.7$ & $<0.7$ \\
VIII & 277071 & - & $<0.7$ & $<0.7$ \\
\hline
\end{tabular}

Notes. The smoothness of the break in the fit using the smooth break between the low- and high-energy spectral index is $2.2 \pm 0.2$.
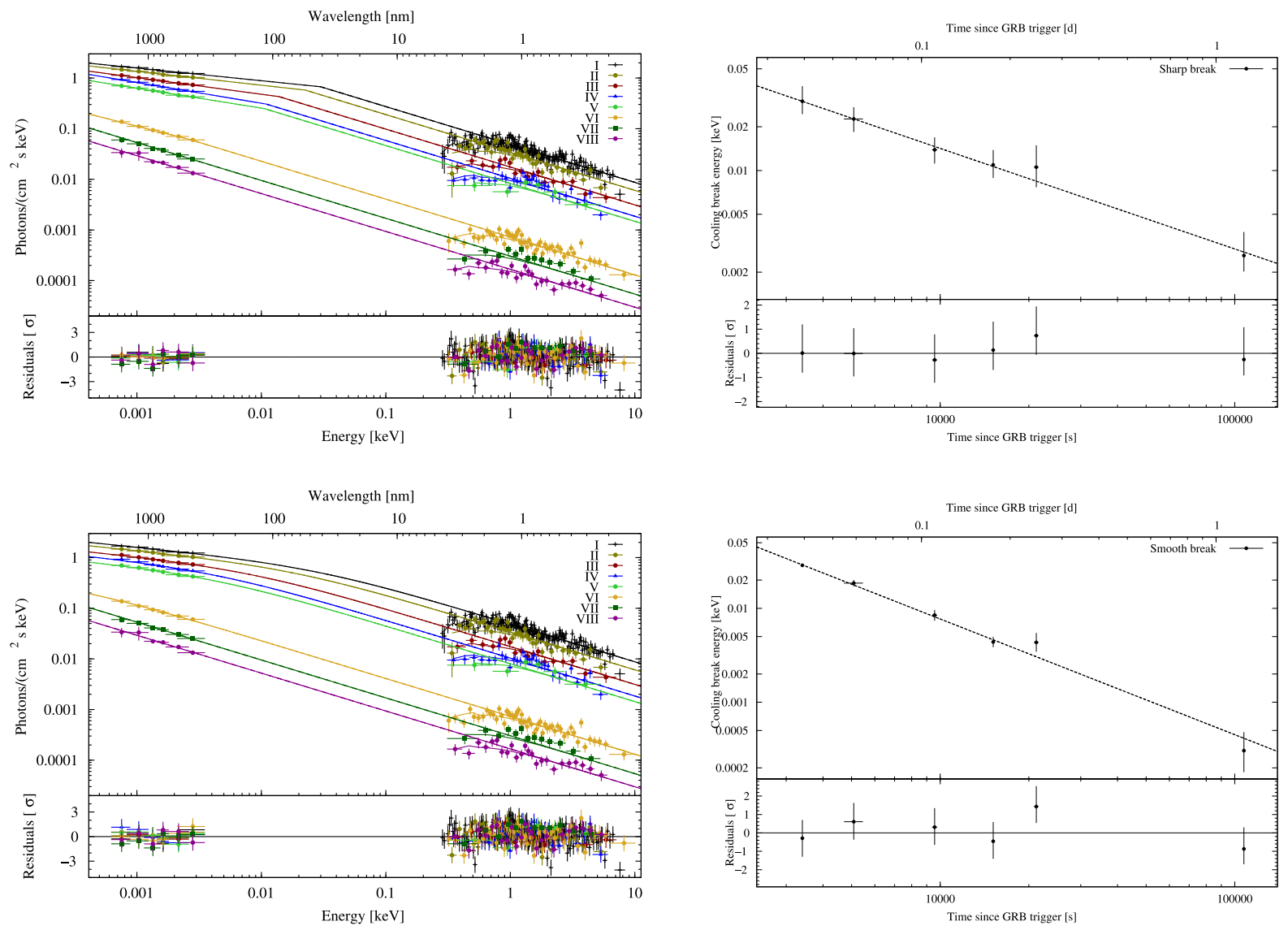

Fig. 5. Broad-band optical/NIR to X-ray SEDs fitted with a broken power-law with the sharp break (top left) and with a broken power-law with the smooth break (bottom left). Best-fit power-law fits to the temporal evolution of the cooling-break energy are shown on the right, resulting from the sharp (top) and the smooth (bottom) broken power-law fits.

Because the fit using the sharp break requires the low-energy spectral index $\beta_{\text {opt }}$ to be time-dependent, we needed a model that would be consistent with constant spectral indices that the theory expects. We therefore also fitted all eight broad-band SEDs simultaneously with two power-laws connected by a smooth break with flux density following

$F_{v} \propto\left[\left(v / v_{\text {break }}\right)^{-s \beta_{1}}+\left(v / v_{\text {break }}\right)^{-s \beta_{2}}\right]^{-1 / s}$,

where $s$ is a parameter that describes the sharpness of the break. Given that the break is far from the X-ray bands, we do not expect the change in the model from a sharp to a smooth break to change the best-fit values of the host absorbing column density $N_{\mathrm{H}}$ nor the high-energy spectral index $\beta_{\mathrm{X}}$. We therefore froze $N_{\mathrm{H}}$ and $\beta_{\mathrm{X}}$ to the best-fit value from the sharp broken power-law fit in order to reduce the number of free parameters in this more complicated model. We fixed the difference in values between low and high energy spectral indices to 0.5 (as predicted for the cooling break by the standard fireball model; Sari et al. 1998). The smoothness of the break was tied between each SED but left free to vary and the break energy was left free to vary completely. The fit (Fig. 5, lower panels) again shows the break moving towards the lower energies but in this case the movement 
A\&A 535, A57 (2011)

Table 5. Secondary standards in the GRB field in the GROND filter bands used for the calibration.

\begin{tabular}{|c|c|c|c|c|c|c|c|}
\hline $\begin{array}{l}\text { Star } \\
\text { number }\end{array}$ & $\begin{array}{c}\text { RA, Dec } \\
{[\mathrm{J} 2000]}\end{array}$ & $\begin{array}{c}g^{\prime} \\
\left(\operatorname{mag}_{\mathrm{AB}}\right)\end{array}$ & $\begin{array}{c}r^{\prime} \\
\left(\operatorname{mag}_{\mathrm{AB}}\right)\end{array}$ & $\begin{array}{c}i^{\prime} \\
\left(\operatorname{mag}_{\mathrm{AB}}\right)\end{array}$ & $\begin{array}{c}z^{\prime} \\
\left(\operatorname{mag}_{\mathrm{AB}}\right)\end{array}$ & $\begin{array}{c}J \\
\left(\operatorname{mag}_{\text {Vega }}\right)\end{array}$ & $\begin{array}{c}H \\
\left(\operatorname{mag}_{\mathrm{Vega}}\right)\end{array}$ \\
\hline 1 & $02: 26: 21.05,-18: 57: 19.1$ & $15.18 \pm 0.03$ & $14.49 \pm 0.03$ & $14.22 \pm 0.03$ & $14.07 \pm 0.03$ & $13.03 \pm 0.05$ & $12.57 \pm 0.05$ \\
\hline 2 & $02: 26: 12.17,-18: 57: 17.6$ & $17.48 \pm 0.03$ & $16.64 \pm 0.03$ & $16.26 \pm 0.03$ & $16.06 \pm 0.03$ & $14.47 \pm 0.05$ & $14.38 \pm 0.05$ \\
\hline 3 & $02: 26: 12.14,-18: 57: 02.9$ & $17.74 \pm 0.03$ & $16.96 \pm 0.03$ & $16.80 \pm 0.03$ & $16.71 \pm 0.03$ & $14.93 \pm 0.05$ & $15.35 \pm 0.05$ \\
\hline 4 & $02: 26: 23.64,-18: 58: 17.8$ & $22.17 \pm 0.03$ & $20.43 \pm 0.03$ & $19.34 \pm 0.03$ & $18.80 \pm 0.03$ & - & - \\
\hline 5 & $02: 26: 25.03,-18: 58: 45.5$ & $20.59 \pm 0.03$ & $19.05 \pm 0.03$ & $18.16 \pm 0.03$ & $17.71 \pm 0.03$ & - & - \\
\hline
\end{tabular}

is faster than with the sharp break and the fit of the energy over time gives a power-law slope of $-1.23 \pm 0.06$.

\subsection{Closure relations}

Using the X-ray light-curve fit and the results from the broadband SEDs, we can test the closure relations (Granot \& Sari 2002; Dai \& Cheng 2001; Zhang \& Mészáros 2004; Racusin et al. 2009) between temporal and spectral indices. The fitderived X-ray spectral index $\beta_{\mathrm{X}}=0.75$ results in a fairly hard power-law index of the electron energy distribution $p=$ $1.50 \pm 0.01$. In the X-rays, the equation (Racusin et al. 2009) for $1<p<2$ and a constant decay in the $v_{\mathrm{X}}>v_{\mathrm{c}}$ regime, where the jet is interacting with a homogeneous interstellar medium (ISM) and is in the slow cooling phase, gives value of $\alpha_{\mathrm{X}}=0.91$ for the spectral index $\beta_{\mathrm{X}}=0.75$ derived from the fits. This value is within $3 \sigma$ of the X-ray light curve pre-break decay slope of $1.02 \pm 0.04$. However, the fast cooling phase in the $v_{\mathrm{X}}>v_{\mathrm{m}}$ regime gives the same value, therefore we cannot distinguish between fast and slow cooling.

The light curve break at X-rays around 33 ks must obviously be due to a different phenomenon than the cooling break, as the latter started already below the X-ray band at $\sim 3 \mathrm{ks}$, and then moved to longer wavelengths. The post-break evolution of the $\mathrm{X}$-ray light curve is best fitted with the equation describing a non-spreading uniform jet in the ISM, which gives $\alpha_{\mathrm{X}}=1.66$, a value consistent within $2 \sigma$ of the fit-derived $1.61 \pm 0.04$. This suggests that, despite the X-ray decay slopes being shallower than the canonical values (Zhang et al. 2006; Nousek et al. 2006), the break in the light curve at around $33 \mathrm{ks}$ represents a jet break (Sari et al. 1999). Such shallow ( $<2$ with high confidence) post-break decay slopes have been seen in multiple wellsampled optical light curves (Zeh et al. 2006). From the time of the break we can estimate the opening angle of the jet to be $\theta \sim 4^{\circ}$ (Burrows \& Racusin 2006), substituting the measured quantities and normalizing to the typical values $n=1 \mathrm{~cm}^{-3}$ and $\eta=0.2$. These values lead to the beaming factor and the true gamma-ray energy release (Frail et al. 2001; Bloom et al. 2003) of $f_{\mathrm{b}}=\left(1-\cos \theta_{\text {jet }}\right)=2.4 \times 10^{-3}$ and $E_{\gamma}=3.9 \times 10^{49} \mathrm{erg}$. For a value of $n=3 \mathrm{~cm}^{-3}$, which is the standard value used for the Ghirlanda relation (Ghirlanda et al. 2007), we get a jet opening angle $\theta \sim 4.9^{\circ}$ and $E_{\gamma}=5.9 \times 10^{49} \mathrm{erg}$. With these values, GRB 091127 lies within the $1 \sigma$ scatter of the Ghirlanda relation.

\section{Discussion}

The high quality of the data allows us to discuss whether any characteristic synchrotron spectral break could be responsible for the break in the afterglow SED of GRB 091127, and to constrain the sharpness of the break.

\subsection{Injection break}

The shape of our broad-band SEDs suggests that the only plausible scenario for the break to be $v_{\mathrm{m}}$ is the fast-cooling case (Sari et al. 1998). According to the equations in Dai \& Cheng (2001), in the case of an ISM medium and for $p=1.5$, the characteristic synchrotron frequency $v_{\mathrm{m}}$ moves towards lower frequencies as $t^{-2.6}$. That is too fast to be consistent with our measurements of the break evolution both for the sharp and the smooth break. The predicted light curve slope of $\alpha=0.25$ before the passage of the injection break is slightly flatter than our early optical slope. But as previously stated, this slope determination is difficult due to the smooth curvature of the early optical/NIR light curve.

However, it is the low-energy spectral slope that is least consistent with the injection break scenario. The SED below $v_{\mathrm{m}}$ is expected to be a power-law with index 0.5 , completely independent of the electron energy distribution $p$. This is not consistent with either the sharp break, where the initial slope is a factor 2 flatter and moreover evolving in time, or the smooth break, where the low-energy slope is 0.25 throughout the observation. While this value was fixed in the smooth-break fit, any steeper low-energy slope makes the fit considerably worse and the initial flat optical/NIR only SEDs impossible to explain. Therefore we argue that the moving break in the afterglow of GRB 091127 cannot be interpreted as the characteristic synchrotron frequency $v_{\mathrm{m}}$.

\subsection{Cooling break}

\subsubsection{Theoretical expectations}

According to theory (Sari et al. 1998; Dai \& Cheng 2001), in case of an ISM circum-burst environment, the cooling break moves towards lower frequencies with time as a power-law with index -0.5 . This is within $2 \sigma$ of the sharp break fits (Fig. 5), where the break moves with index $-0.69 \pm 0.10$. However, the sharp-break fit requires temporal change of the low-energy spectral index. This is inconsistent with the fireball model, where the difference between low- and high-energy spectral indices below and above the cooling frequency is constant and $\Delta \beta=0.5$.

To satisfy the condition of a constant $\Delta \beta$, we fitted the SEDs with a smooth break, that can gradually change the spectral index of the data, which occupies a sufficiently narrow portion of the spectra (in this case optical/NIR wavelengths) to not show evidence for inherent curvature. The smooth-break fit therefore allows both low- and high-energy indices to remain constant, while changing the spectral index fit to GROND data with time, as the break crosses the optical bands (Fig. 4). Before any further discussion, we need to address the question of the physical plausibility of the smooth break.

When we examined the SEDs from studies of large GRB samples (Greiner et al. 2011; Schady et al. 2007, 2010; Nardini et al. 2006; Starling et al. 2007), we see that they are well fitted with a sharp cooling break (where the break is plausible). 
This simplistic choice works well for sample studies where it is difficult to distinguish between a sharp and a smooth shape of the break either because the break is far enough from the measured data or because the data lack sufficient quality to constrain the smoothness parameter, but can fail in cases like GRB 091127, where extremely large multi-color data sets are available. Although previous studies did not require more complex models, Granot \& Sari (2002) calculated that the powerlaws in the afterglow spectra are indeed connected by smooth breaks. The theoretical smoothness of the cooling break is $1.15-0.06 p=1.06$ for $p=2 \times \beta_{\mathrm{X}}=1.5$. This is roughly a factor of 2 less (i.e., smoother) than our fit-derived smoothness of $2.2 \pm 0.2$.

The significant inconsistency, however, is related to the speed of the cooling break, which in the smooth fit moves with an index $-1.23 \pm 0.06$, a value much higher than the expected -0.5 . Similar to the value of $-1.00 \pm 0.14$ derived for the cooling break movement reported by Racusin et al. (2009), it would require that we abandon some simplifications often assumed in the simplest formulations of the fireball model. The flux evolution for adiabatic slow cooling in this synchrotron emission theory is described by Eq. (8) in Sari et al. (1998) and for convenience we report it here as

$F_{v}= \begin{cases}\left(v / v_{\mathrm{m}}\right)^{-(p-1) / 2} F_{v, \max }, & v_{\mathrm{c}}>v>v_{\mathrm{m}}, \\ \left(v_{\mathrm{c}} / v_{\mathrm{m}}\right)^{-(p-1) / 2}\left(v / v_{\mathrm{c}}\right)^{-p / 2} F_{v, \max }, & v>v_{\mathrm{c}},\end{cases}$

where the break frequencies for the case of $p<2$ can be calculated from Dai \& Cheng (2001) and Chevalier \& Li (2000) to be

$$
\begin{aligned}
& v_{\mathrm{c}} \propto \epsilon_{\mathrm{B}}^{-3 / 2} E_{\mathrm{iso}}^{-1 / 2} t^{-1 / 2}, \\
& v_{\mathrm{m}} \propto \epsilon_{\mathrm{B}}^{1 / 2(p-1)} \epsilon_{e}^{2 /(p-1)} E_{\text {iso }}^{p+2 / 8(p-1)} t^{-3(p+2) / 8(p-1)}, \\
& F_{v, \max } \propto \epsilon_{\mathrm{B}}^{1 / 2} E_{\mathrm{iso}},
\end{aligned}
$$

where $t$ is the time since the GRB trigger, $E_{\text {iso }}$ is the isotropic energy of the GRB, $\epsilon_{\mathrm{B}}$ is the fraction of the energy carried by the magnetic field and $\epsilon_{\mathrm{e}}$ the fraction of the energy in electrons. In the standard fireball model, all parameters are constant in time and the density in the ISM is homogeneous. For the cooling break speed to be consistent with our measurements, one of the parameters $\epsilon_{\mathrm{B}}$ and $E_{\text {iso }}$ (or a combination of them) must evolve with time. Using Eqs. (2) and (3), we can easily examine cases where each of these parameters evolves separately and model the impact of such an evolution on the resulting afterglow flux.

\subsubsection{Theoretical implications}

To obtain the measured cooling break speed of $t^{-1.23 \pm 0.06}$ we need one of the parameters (we treat them separately for simplicity) to add $t^{-0.73 \pm 0.06}$ to the theoretical speed of $t^{-0.5}$. As we can see from the Eq. (2), the change of the flux evolution before and after the cooling break passage is proportional to the cooling break frequency evolution as $v_{\mathrm{c}}^{0.5}$. This means that the cooling break that is faster by a factor of $t^{-0.73 \pm 0.06}$ would add $\Delta \alpha=0.37 \pm 0.03$ to the standard change of the temporal index of $\Delta \alpha=0.25$ (Sari et al. 1998) caused by the cooling brake passage.

As we already stated, the early optical/NIR slope is difficult to obtain. However, we can estimate it by calculating the weighted mean of the values of the optical/NIR parameter $\alpha_{1}$ in Table 1. This results in a decay index of $\alpha=0.38$ before the jet break at around $33 \mathrm{ks}$. If we assume this to be the decay index before the cooling break passage, and we take the X-ray pre-jetbreak temporal slope of $\alpha=1.02 \pm 0.04$ to be the one after the cooling break passage, we get a very good (within $1 \sigma$ ) consistency with our calculated $\Delta \alpha=0.62 \pm 0.03$. While the amount by which the light-curve steepens is only dependent on the speed of the cooling break and not on which parameter causes it, the flux evolution and therefore the decay index itself before and after the cooling break passage depends strongly on which parameter we let evolve in time. Using Eqs. (2) and (3), we can calculate how the time evolution of the flux density depends on these parameters for $p<2$ (for $p>2$ see Eqs. (B7) and (B8) in Panaitescu \& Kumar 2000). We calculate

$F_{v} \propto \begin{cases}E^{(p+18) / 16} \epsilon_{\mathrm{B}}^{3 / 4} t^{-3(p+2) / 16}, & v_{\mathrm{c}}>v>v_{\mathrm{m}}, \\ E^{(p+14) / 16} t^{-(3 p+10) / 16}, & v>v_{\mathrm{c}} .\end{cases}$

Letting the isotropic energy vary in time results in $F_{v} \propto$ $E^{(p+18) / 16}$ for $v<v_{\mathrm{c}}$ and $F_{v} \propto E^{(p+14) / 16}$ for $v>v_{\mathrm{c}}$. In this case the increased speed of the cooling break is the result of the isotropic energy which increases in time as $t^{1.46}$. This dependence using the fit-derived $p=1.5$ decreases the temporal index before and after the cooling break passage by 1.78 and 1.41 respectively. Such extreme flattening of the light curve would mean that without the energy injection the decay slope before the jet break would be $\alpha_{1}=1.02+1.41=2.4$ and the late temporal slope after the jet break $\alpha_{2}=1.61+1.41=3.0$, values which are unusually steep for a GRB afterglow (Racusin et al. 2009). The energy $E_{\text {iso }}$ is directly dependent on the energy injection and indirectly on the density profile around the burst and we can examine the influence of the time evolution of these parameters on the energy using equations from Sari \& Mészáros (2000).

The density profile of the medium can be calculated from the cooling-break temporal exponent using equations in Table 1 of Sari \& Mészáros (2000). There $v_{\mathrm{c}} \propto t^{(3 g-4) / 2(4-g)}$, where $g$ is the power-law index of the external density profile $n \propto r^{-g}$. The same approach was used by Racusin et al. (2009) for GRB 080319B where the cooling break speed of $t^{-1}$ results in the steep density profile $n \propto r^{4}$, which requires the existence of a complex medium with a density enhancement. However, our cooling break speed of $t^{-1.23}$ implies an implausibly steep density profile of $n \propto r^{11}$, which would be very difficult to defend physically and support observationally.

Using Eq. (11) in Sari et al. (1998) for the cooling break frequency and assuming typical values of $n_{1}=1$ and $\epsilon_{\mathrm{B}}=0.01$, we can calculate the isotropic energy of the burst at times corresponding to the first (SED I) and the last (SED VI) point where we measure the position of the cooling break using the smooth break fit. The best-fit parameters in Table 2 give $E_{52} \sim 3.8$ at $t=3.4 \mathrm{ks}$ and $E_{52} \sim 1080$ at $t=107.4 \mathrm{ks}$. The increasing energy of GRBs can possibly be explained by refreshed shocks, where the central engine ejects shells with a range of Lorentz factors. When the slower material catches up with the decelerating ejecta, it re-energizes it (Sari \& Mészáros 2000). However, assuming a constant density profile, this scenario requires extreme energy injection, leading to an injection parameter $s=8.6$ (see Table 1 in Sari \& Mészáros 2000). Such a scenario is very unlikely, as it would require the initial low-energy ejecta to be re-energized by a very large amount of energy stored in slowly moving material. It would also require a gradual and continuous energy injection over the time of our light curve coverage, i.e. $\sim 10^{6} \mathrm{~s}$, a scenario which so far has never been advocated. We therefore also consider a change of energy input an unlikely explanation for the temporal behavior of GRB 091127. 
The last option is to let the microphysical parameter $\epsilon_{\mathrm{B}}$ vary in time. To be consistent with our measurement of the cooling break speed, the fraction of energy in the magnetic field would have to rise in time as $\epsilon_{\mathrm{B}} \propto t^{0.49}$. Such an evolution would influence the flux as $F_{v} \propto \epsilon_{\mathrm{B}}^{3 / 4}$ for $v<v_{\mathrm{c}}$ while the flux density is independent of $\epsilon_{\mathrm{B}}$ for $v>v_{\mathrm{c}}$. Therefore the temporal index before the cooling break passage would decrease by 0.37 on top of the theoretical flux density evolution. This flattening of the temporal index in the $v<v_{\mathrm{c}}$ regime would explain the early shallow optical/NIR decay, while the late data after the jet break would not be influenced by an evolving $\epsilon_{\mathrm{B}}$. We can again use Eq. (11) in Sari et al. (1998) to estimate the value of $\epsilon_{\mathrm{B}}$, assuming $E_{52}=1.6$ and $n_{1}=1$. The calculation results in $\epsilon_{\mathrm{B}}=0.013$ at $t=3.4 \mathrm{ks}$, a value consistent with standard models, and $\epsilon_{\mathrm{B}}=0.088$ at $t=107.4 \mathrm{ks}$.

There is a growing number of studies which have modelled broad-band GRB light curves, and these have yielded results for $\epsilon_{\mathrm{B}}$ which span several orders of magnitude between different GRBs, with values from $\sim 10^{-5}$ to $\sim 10^{-1}$ (Panaitescu \& Kumar 2001, 2002; Yost et al. 2003), raising questions whether the assumption of $\epsilon_{\mathrm{B}}$ being constant in the simplest fireball model is consistent with the observations. Lately, the idea of $\epsilon_{\mathrm{B}}$ increasing in time as a power-law has been discussed and is receiving increasing support from observational data (e.g., Panaitescu et al. 2006; Kong et al. 2010). There is also the possibility that all the parameters that influence the cooling frequency vary in time simultaneously. However, it would require more sophisticated theoretical work to derive some estimates or constraints on the ratios between them; our data cannot provide such constraints.

The discussion so far was based on the assumption that the environment around the burst is the undisturbed ISM, i.e. the radial density profile is constant. While this assumption is supported by the closure relations and the direction of the spectral break, we must consider also the possibility that the circumburst density has a wind profile. In that case we would expect from the theory the cooling break to move towards shorter wavelengths as $v_{\mathrm{c}} \propto t^{0.5}$. To be consistent with our measurement of $t^{-1.23}$, the parameters in Eq. (3) would have to increase in time so rapidly, that they would effectively reverse the direction of the cooling break movement. Given that we concluded that the time evolution of parameter $E$ is too dramatic in the ISM scenario, the even more rapid increase required here is more unlikely. To reverse the cooling break movement, $\epsilon_{\mathrm{B}}$ would have to increase its time evolution to $t^{1.15}$. While we cannot completely rule out this option due to the inability to compute the exact values of $\epsilon_{\mathrm{B}}$ in evolving density, we believe that such rapid time evolution would be difficult to defend against the ISM scenario.

\section{Conclusions}

Since the launch of the Swift satellite, there is growing evidence that the radiative mechanism responsible for the optical to X-ray GRB emission is not as simple and well understood as previously believed. The growing number of well-sampled data sets (Covino et al. 2010; Guidorzi et al. 2009; Thöne et al. 2010; Filgas et al. 2011) is beginning to place strong constraints on the fireball model and possible alternatives (e.g., Dar \& De Rujula 2000; Dado et al. 2009). Most GRBs have complex light curves, for which the optical and X-ray emission are seemingly decoupled, thus providing an indication that they are produced by different mechanisms. The afterglow of GRB 091127 is one of the few examples in which the light-curve evolution in the optical/NIR and X-ray wavelengths is well represented by a broken power-law and, in addition, both light curves show a break at roughly the same time and similar decay slopes after that break. This observational evidence, together with the fact that the optical/NIR to X-ray SED at late times is well represented by a single component, leads us to an assumption that the emission in both energy bands has been produced by the same radiative mechanism and that this mechanism could be the standard external shock synchrotron radiation.

We observe a clear break in the light curve at around $33 \mathrm{ks}$, which we interpret as a jet break, based on the fact that it is achromatic and the post-break evolution of all bands is similar. The GROND SEDs show a strong color evolution with the optical/NIR spectral index rising from roughly 0.25 to 0.75 , while the X-ray spectral slope stays constant. The broad-band NIR to X-ray SEDs were fitted with a broken power-law with the break moving in time towards larger wavelengths. Because the difference between the low- and high-energy spectral index reaches 0.5 asymptotically, we interpret the spectral break as the cooling break, decreasing in energy with time, as the forward shock moves into an ISM-like circumburst medium. Since it takes almost all the follow-up time for the optical/NIR spectral slope to gradually steepen from the initial value to the value consistent with the X-ray spectral index, we conclude that the cooling break is very smooth in frequency space.

The measured cooling break speed of $v_{\mathrm{c}} \propto t^{-1.23 \pm 0.06}$ is faster than expected for a shock evolving in a constant density medium and requires that one of the parameters that influence the afterglow flux density evolves with time. We conclude that the required changes in the energy release $E_{\text {iso }}$ alone would be too dramatic to be physically plausible and that the most feasible explanation is the evolution of microphysical parameters. Assuming $\epsilon_{\mathrm{B}}$ (the fraction of the energy carried by the magnetic field) to be the only varying parameter, then during the time interval that we measure the position of the cooling break, between 3 and $107 \mathrm{ks}$, it would rise in time as $\epsilon_{\mathrm{B}} \propto t^{0.49}$, and would reach values of 0.01 and 0.09 at those times, respectively.

Currently, a complete understanding of the microphysical processes is still lacking. Nonetheless, data from instruments like Swift and GROND can shed some light on the shock physics. A larger study of the observational data of bursts similar to GRB 091127 is necessary to investigate how commonly such changes in $\epsilon_{\mathrm{B}}$ occur in GRB afterglows. Theoretical studies would be warranted to investigate effects which would change $\epsilon_{\mathrm{B}}$ as the fireball expands into its surrounding environment.

Acknowledgements. We thank the anonymous referee for constructive comments that helped to improve the paper. Part of the funding for GROND (both hardware as well as personnel) was generously granted from the LeibnizPrize to Prof. G. Hasinger (DFG grant HA 1850/28-1). This work made use of data supplied by the UK Swift Science Data Centre at the University of Leicester. T.K. acknowledges support by the DFG cluster of excellence Origin and Structure of the Universe. T.K. acknowledges support by the European Commission under the Marie Curie Intra-European Fellowship Programme. The Dark Cosmology Centre is funded by the Danish National Research Foundation. F.O.E. acknowledges funding of his Ph.D. through the Deutscher Akademischer Austausch-Dienst (DAAD). S.K., D.A.K. and A.N.G. acknowledge support by DFG grant K1 766/16-1. A.R. acknowledges support from the BLANCEFLOR Boncompagni-Ludovisi, née Bildt foundation. M.N. acknowledges support by DFG grant SA 2001/2-1. P.S. acknowledges support by DFG grant SA 2001/1-1. A.C.U., A.N.G., D.A.K. and A.R. are grateful for travel funding support through MPE.

\section{References}

Amati, L., Frontera, F., Tavani, M., et al. 2002, A\&A, 390, 81

Band, D., Matteson, J., Ford, L., et al. 1993, ApJ, 413, 281

Barthelmy, S. D., Barbier, L. M., Cummings, J. R., et al. 2005, Space Sci. Rev., 120,143 
Berger, E., Chornock, R., Holmes, T. R., et al. 2011, ApJ, submitted [arXiv: 1106.3073]

Beuermann, K., Hessman, F. V., Reinsch, K., et al. 1999, A\&A, 352, L26

Bloom, J. S., Frail, D. A., \& Kulkarni, S. R. 2003, ApJ, 594, 674

Blustin, A. J., Band, D., Barthelmy, S., et al. 2006, ApJ, 637, 901

Burrows, D. N., \& Racusin, J. 2006, Nuovo Cimento B Serie, 121, 1273

Burrows, D. N., Hill, J. E., Nousek, J. A., et al. 2005, Space Sci. Rev., 120, 165

Chevalier, R. A., \& Li, Z.-Y. 2000, ApJ, 536, 195

Cobb, B. E., Bloom, J. S., Perley, D. A., et al. 2010, ApJ, 718, L150

Covino, S., Campana, S., Conciatore, M. L., et al. 2010, A\&A, 521, A53

Cucchiara, A., Fox, D., Levan, A., et al. 2009, GRB Coordinates Network, Circular Service, 10202, 1

Dado, S., Dar, A., \& De Rújula, A. 2009, ApJ, 696, 994

Dai, Z. G., \& Cheng, K. S. 2001, ApJ, 558, L109

Dar, A., \& De Rujula, A. 2000, unpublished [arXiv: astro-ph/0008474]

Evans, P. A., Beardmore, A. P., Page, K. L., et al. 2007, A\&A, 469, 379

Evans, P. A., Beardmore, A. P., Page, K. L., et al. 2009a, MNRAS, 397, 1177

Evans, P. A., Page, K. L., \& Troja, E. 2009b, GRB Coordinates Network, Circular Service, 10201, 1

Filgas, R., Krühler, T., Greiner, J., et al. 2011, A\&A, 526, A113

Frail, D. A., Kulkarni, S. R., Sari, R., et al. 2001, ApJ, 562, L55

Ghirlanda, G., Nava, L., Ghisellini, G., et al. 2007, A\&A, 466, 127

Gehrels, N., Chincarini, G., Giommi, P., et al. 2004, ApJ, 611, 1005

Golenetskii, S., Aptekar, R., Frederiks, D., et al. 2009, GRB Coordinates

Network, Circular Service, 10209, 1

Granot, J., \& Sari, R. 2002, ApJ, 568, 820

Greiner, J., Bornemann, W., Clemens, C., et al. 2007, The Messenger, 130, 12

Greiner, J., Bornemann, W., Clemens, C., et al. 2008, PASP, 120, 405

Greiner, J., Krühler, T., Fynbo, J. P. U., et al. 2009a, ApJ, 693, 1610

Greiner, J., Krühler, T., McBreen, S., et al. 2009b, ApJ, 693, 1912

Greiner, J., Krühler, T., Klose, S., et al. 2011, A\&A, 526, A30

Grupe, D., Burrows, D. N., Wu, X. F., et al. 2010, ApJ, 711, 1008

Guidorzi, C., Clemens, C., Kobayashi, S., et al. 2009, A\&A, 499, 439

Huang, K. Y., Urata, Y., Filippenko, A. V., et al. 2005, ApJ, 628, L93

Immler, S., \& Troja, E. 2009, GRB Coordinates Network, Circular Service, 10199, 1

Kalberla, P. M. W., Burton, W. B., Hartmann, D., et al. 2005, A\&A, 440, 775

Kann, D. A., Klose, S., Zhang, B., et al. 2010, ApJ, 720, 1513

Komatsu, E., Dunkley, J., Nolta, M. R., et al. 2009, ApJS, 180, 330

Kong, S. W., Wong, A. Y. L., Huang, Y. F., et al. 2010, MNRAS, 402, 409

Krühler, T., Küpcü Yoldaş, A., Greiner, J., et al. 2008, ApJ, 685, 376

Krühler, T., Greiner, J., Afonso, P., et al. 2009, A\&A, 508, 593

Krühler, T., Schady, P., Greiner, J., et al. 2011a, A\&A, 526, A153

Krühler, T., Greiner, J., Schady, P., et al. 2011b, A\&A, 534, A108

Kumar, P., \& Panaitescu, A. 2000, ApJ, 541, L9

Küpcü Yoldaş, A., Krühler, T., Greiner, J., et al. 2008, Am. Inst. Phys. Conf. Ser., 1000, 227

Küpcü Yoldaş, A., Greiner, J., Klose, S., et al. 2010, A\&A, 515, L2

Lipkin, Y. M., Ofek, E. O., Gal-Yam, A., et al. 2004, ApJ, 606, 381
Monet, D. G., Levine, S. E., Canzian, B., et al. 2003, AJ, 125, 984

Mészáros, P. 2002, ARA\&A, 40, 137

Mészáros, P., \& Rees, M. J. 1997, ApJ, 476, 232

Nardini, M., Ghisellini, G., Ghirlanda, G., et al. 2006, A\&A, 451, 821

Nardini, M., Greiner, J., Krühler, T., et al. 2011, A\&A, 531, A39

Nousek, J. A., Kouveliotou, C., Grupe, D., et al. 2006, ApJ, 642, 389

Panaitescu, A., \& Kumar, P. 2000, ApJ, 543, 66

Panaitescu, A., \& Kumar, P. 2001, ApJ, 560, L49

Panaitescu, A., \& Kumar, P. 2002, ApJ, 571, 779

Panaitescu, A., Mészáros, P., Burrows, D., et al. 2006, MNRAS, 369, 2059

Pei, Y. C. 1992, ApJ, 395, 130

Piran, T. 1999, Phys. Rep., 314, 575

Racusin, J. L., Karpov, S. V., Sokolowski, M., et al. 2008, Nature, 455, 183

Racusin, J. L., Liang, E. W., Burrows, D. N., et al. 2009, ApJ, 698, 43

Roming, P. W. A., Kennedy, T. E., Mason, K. O., et al. 2005, Space Sci. Rev., 120,95

Sari, R., \& Mészáros, P. 2000, ApJ, 535, L33

Sari, R., Piran, T., \& Narayan, R. 1998, ApJ, 497, L17

Sari, R., Piran, T., \& Halpern, J. P. 1999, ApJ, 519, L17

Sato, R., Kawai, N., Suzuki, M., et al. 2004, Gamma-Ray Bursts: 30 Years of Discovery, 727, 307

Schady, P., Mason, K. O., Page, M. J., et al. 2007, MNRAS, 377, 273

Schady, P., Page, M. J., Oates, S. R., et al. 2010, MNRAS, 401, 2773

Schlegel, D. J., Finkbeiner, D. P., \& Davis, M. 1998, ApJ, 500, 525

Skrutskie, M. F., Cutri, R. M., Stiening, R., et al. 2006, AJ, 131, 1163

Smith, J. A., Tucker, D. L., Kent, S., et al. 2002, AJ, 123, 2121

Stamatikos, M., Barthelmy, S. D., Baumgartner, W. H., et al. 2009, GRB Coordinates Network, Circular Service, 10197, 1

Starling, R. L. C., Wijers, R. A. M. J., Wiersema, K., et al. 2007, ApJ, 661, 787

Thöne, C. C., Goldoni, P., Covino, S., et al. 2009, GRB Coordinates Network, Circular Service, 10233, 1

Thöne, C. C., Kann, D. A., Jóhannesson, G., et al. 2010, A\&A, 523, A70

Tody, D. 1993, in Astronomical Data Analysis Software and Systems II, ed. R. J. Hanisch, R. J. V. Brissenden, \& J. Bames, San Francisco, CA, ASP Conf Ser., 52,173

Torii, K., Fukazawa, Y., \& Tsunemi, H. 2005, A\&A, 437, L23

Troja, E., Barthelmy, S. D., Baumgartner, W. H., et al. 2009, GRB Coordinates Network, Circular Service, 10191, 1

Uemura, M., Kato, T., Ishioka, R., et al. 2003, Nature, 423, 843

de Ugarte Postigo, A., Castro-Tirado, A. J., Gorosabel, J., et al. 2005, A\&A, 443, 841

Updike, A., Rossi, A., Rau, A., et al. 2009, GRB Coordinates Network, Circular Service, 10195, 1

Vergani, S. D., Flores, H., Covino, S., et al. 2011, A\&A, in press, DOI: $10.1051 / 0004-6361 / 201117726$

Yost, S. A., Harrison, F. A., Sari, R., et al. 2003, ApJ, 597, 459

Yost, S. A., Alatalo, K., Rykoff, E. S., et al. 2006, ApJ, 636, 959

Zeh, A., Klose, S., \& Kann, D. A. 2006, ApJ, 637, 889

Zhang, B., \& Mészáros, P. 2004, Int. J. Mod. Phys. A, 19, 2385

Zhang, B., Fan, Y. Z., Dyks, J., et al. 2006, ApJ, 642, 354 